OPEN ACCESS

Edited by:

Adelaida María AM Castro Sánchez, University of Almería, Spain

Reviewed by:

Gioia Mura,

University of Cagliari, Italy

Giorgio Sandrini,

University of Pavia, Italy

${ }^{*}$ Correspondence: Danielle T. Felsberg

dtfelsbe@uncg.edu

Specialty section:

This article was submitted to Movement Science and Sport

Psychology,

a section of the journal

Frontiers in Psychology

Received: 30 January 2019 Accepted: 12 April 2019 Published: 08 May 2019

Citation:

Felsberg DT, Maher JP and Rhea CK (2019) The State of Behavior Change Techniques in Virtual Reality Rehabilitation of Neurologic Populations. Front. Psychol. 10:979. doi: 10.3389/fpsyg.2019.00979

\section{The State of Behavior Change Techniques in Virtual Reality Rehabilitation of Neurologic Populations}

\author{
Danielle T. Felsberg ${ }^{1 *}$, Jaclyn P. Maher ${ }^{2}$ and Christopher K. Rhea ${ }^{1}$ \\ ${ }^{1}$ Virtual Environment for Assessment and Rehabilitation (VEAR) Lab, Department of Kinesiology, University of North Carolina \\ at Greensboro, Greensboro, NC, United States, ${ }^{2}$ Physical Activity and Lifetime Wellness Lab, Department of Kinesiology, \\ University of North Carolina at Greensboro, Greensboro, NC, United States
}

Background: Neurologic rehabilitation aims to restore function, address barriers to activity, and improve quality of life in those with injury to the nervous system. Virtual reality (VR) has emerged as a useful tool to enhance neurorehabilitation interventions and outcomes. However, the manner in which VR-based neurorehabilitation has been manipulated to optimize outcomes using theory-based frameworks has not been documented. Behavior Change Techniques (BCTs) are described as the smallest active ingredient in an intervention aimed to change behavior via theoretically-proposed pathways. The purpose of this review was to investigate the ways VR is being used in neurorehabilitation to improve upright mobility, and systematically code those VR interventions for active BCTs.

Methods: Keyword searches were performed using database searches of PubMed, SPORTDiscus, and psycINFO. The search yielded 32 studies for inclusion. Coding for BCTs was conducted using the Behavior Change Techniques Taxonomy v1 (BCTTv1).

Results: Behavioral Practice, Graded Tasks, Biofeedback, and Explicit Feedback were the most commonly used BCTs. All studies reported improvements in motor performance outcomes. However, none of the studies investigated the efficacy of each component of their VR intervention making it difficult to point to the most effective components of VR interventions overall.

Conclusions: This review suggests that investigation into the specific components of VR interventions, along with purposeful implementation and reporting of BCTs will help improve understanding of the efficacy of VR as a neurorehabilitation tool. Future research could benefit from incorporating BCTs into the design process of VR interventions to produce optimal rehabilitation potential.

Keywords: virtual reality, neurorehabilitation, gait, mobility, motor performance, behavior change techniques 


\section{INTRODUCTION}

\section{Rationale}

Virtual reality (VR) is an artificial environment containing sensory information (typically in the visual and/or auditory domains) that allows a natural expression of behaviors to emerge (Steinicke et al., 2013). There has been a wide range of contexts to which VR has been applied, including, but not limited to therapeutic programs addressing PTSD, anxiety, phobias, schizophrenia, ADHD, autism, and pain management (Freeman, 2008; Parsons and Rizzo, 2008; Li et al., 2011; Wang and Reid, 2011; Gonçalves et al., 2012; Opriş et al., 2012; Kandalaft et al., 2013). However, its usage in the physical rehabilitation of neurologic populations is relatively new and has grown in recent years (Darekar et al., 2015; Teo et al., 2016; Howard, 2017; Aida et al., 2018; Porras et al., 2018). VR in rehabilitation can take many forms, and is constantly evolving as the field and technology progresses (Kiefer et al., 2013). VR interventions can occur on a continuum of least (or non-) immersive to fully immersive. Changes in the components of the VR design can improve the level of immersion of the experience by the user (Patel et al., 2006). These components include: visual field continuity, interactivity, level and type of feedback, narrative engagement, user freedom, and visual conformity. With large developments in technology, the use of VR has increasingly emerged as a useful tool to enhance physical rehabilitation interventions and outcomes (Ravi et al., 2017; Casuso-Holgado et al., 2018; Porras et al., 2018).

Physical rehabilitation aims to restore function and improve quality of life in those with disability or injury. More specifically, neurologic physical rehabilitation is a specialized area of practice assessing and treating people with injury to the nervous system. For this population, barriers to physical activity include fear of falling, poor outcome expectations, and lack of social support (Mulligan et al., 2012; Ellis et al., 2013). Rehabilitation can help address these barriers and improve function, independence, and quality of life. VR has been shown to be an especially promising tool in rehabilitation to optimize mobility, as it allows patients to actively participate and interact in a dynamic environment (Keshner and Fung, 2017; LoJacono et al., 2017; Rhea and Kuznetsov, 2017; Porras et al., 2018). Furthermore, therapists can individualize VR interventions to best suit their intended outcomes and assess resultant behaviors.

In a recent systematic review and meta-analysis, VR interventions were shown to be more effective at improving motor outcomes than more traditional real-world interventions (Howard, 2017). Rehabilitation interventions, including gait training, used in combination with VR can provide an enriching and optimally stimulating environment (Molina et al., 2014). Zimmerli et al. (2013) outlined five critical components to create an ideally stimulating and optimally engaging VR task. These components are adaptability, explicit feedback, task goals, interactivity, and the option of added competition. For example, an adaptable VR task would be able to scale the difficulty of the task in real-time to adjust to the user's individual needs. Explicit feedback could be given through visual or auditory cues which allow the user to know if they are performing the task correctly, and task goals can be set at the beginning of the VR task. Interactivity speaks to the synchronicity of the user's behavior in the real-world to the outcome in the virtual world. A VR task with high interactivity could have an avatar, or similar representation of the user, which performs actions in the VR in concert with the user's movements in the real-world. Adding the option of competition to a VR would allow the user to perform the task in the VR while competing against a virtual opponent.

Currently, it is not clear the degree to which researchers are incorporating these five components in the design of VR interventions for motor rehabilitation. Moreover, while many of the aforementioned studies have used motor learning principles to design their VR rehabilitation program, adopting only principles of change in the motor domain may limit the adoption and effectiveness of the VR program. A more holistic approach would be to re-conceptualize VR rehabilitation programs in terms of a theoretically-informed framework that describes behavior change using a multi-domain perspective. One such framework applicable in this context is a taxonomy of Behavior Change Techniques (BCTs), which describes the "active ingredients" across a variety of domains needed to change behavior. A taxonomy linking theoretical constructs to BCT components was first developed by Abraham and Miche (2008) and subsequently updated to the "BCT Taxonomy v1" (Abraham and Miche, 2008; Michie et al., 2008, 2013). Although this taxonomy is relatively new, it has already been adopted across a variety of fields in which a change in behavior is desired (Webb et al., 2010; Bird et al., 2013; Conroy et al., 2014; Lyons et al., 2014). However, we are unaware of any papers that have applied this taxonomy of BCTs to examine components of neurologic rehabilitation in VRbased interventions. Systematically identifying BCTs could help characterize the components of past VR research that has led to the most desirable outcomes, providing a roadmap for future researchers and clinicians who wish to use VR as a tool to enhance physical rehabilitation.

\section{Objectives}

The purpose of this review is two-fold: (1) to investigate the ways VR has been used in neurologic rehabilitation to improve motor performance and (2) to systematically code and analyze the state of BCTs in VR neurologic rehabilitation interventions.

\section{Research Question}

The current state of VR interventions to promote improvements in gait and upright mobility in those with neurologic deficits is constantly evolving. Specifically, how current VR interventions are incorporating BCTs to create a more holistic intervention to address mobility deficits is unclear.

\section{METHODS}

\section{Study Design}

This study was designed to be a systematic review of the current literature investigating the role of VR interventions to improve gait and upright mobility in participants with neurologic deficits across the lifespan. The articles selected for inclusion were coded 
using the Behavior Change Technique Taxonomy v1 (BCTTv1) by two independent coders. One coder was an expert in BCTs, and the other had expertise with VR interventions for motor rehabilitation. First, both coders independently categorized the initial thirty-percent of included articles, then met to come to a consensus on use of specific BCTs for final coding of all remaining articles. Disagreements between coders were resolved through discussion following independent coding of all articles.

\section{Participants, Interventions, Comparators}

Articles were chosen for review if the study specifically investigated the effects of a VR intervention on an upright mobility-related outcome. Once studies were selected, all VR interventions were coded using the BCTTv1. The BCCTv1 was created to code BCTs and develop a common language to analyze and replicate interventions in both research and practical settings (Michie et al., 2013). It is a structured taxonomy of 93 BCTs and offers a reliable method for identifying and analyzing the BCT in an intervention (Michie et al., 2013). Due to the objective of this study, only the VR portion of the intervention of each study was coded using this taxonomy.

\section{Systematic Review Protocol}

The systematic review protocol guidelines described by the Preferred Reporting Items for Systematic Reviews and Metaanalysis (PRISMA) were adopted and applied to this review (Moher et al., 2015). Keyword searches for two of the databases were initially performed from October 2017 through November 2017. An additional keyword search was conducted in a third database in October 2018. Articles were screened based on the specific inclusion and exclusion criteria outlined in the following data extraction section. Articles meeting the initial criteria were kept for further full-text assessment of eligibility. Through full-text assessment of each article, articles were excluded using extensive exclusion criteria to find articles for final qualitative synthesis.

\section{Search Strategy}

Keyword searches were performed using database searches of PubMed, SPORTDiscus, and psycINFO. The search parameters used included all possible combinations of (1) "virtual reality" (2) "physical therapy," "physiotherapy," or "rehabilitation" and (3) "gait," "ambulation," "mobility," or "motor performance." The specific search algorithm is provided in Figure 1.

\section{Study Selection}

Once duplicates were removed, studies were excluded through the screening process if they were a systematic review or metaanalysis, a non-research text (editorial, commentary, etc.), did not have full-text available, or were a study protocol, pilot or case study. Additionally, studies were excluded if they did not use neurologic populations, an upright mobility task, or focused on the upper extremity. Following screening, all full-text articles were assessed for eligibility. Studies were selected for inclusion if they were investigating the role of VR in gait or upright mobility outcomes of neurologic populations. Studies were excluded if they did not use VR, were not directly assessing the relationship between VR and motor outcomes, used vestibular populations, or employed an adjunct therapy (i.e., transcranial magnetic stimulation).

\section{Data Sources, Studies Sections, and Data Extraction}

Once the study selection process was completed, each article was reviewed to locate the section which detailed the VR intervention being used in the study. Only the section of the article regarding the VR intervention was used for BCT coding purposes. Other data (sample size, study design, and VR technology used, etc.) was extracted to synthesize the overall state of VR interventions aimed to address mobility deficits in neurologic populations by full-text review of the articles.

\section{Data Analysis}

Following study selection and identification of the VR intervention sections of each included article, both coders independently coded these sections using the BCTTv1. Given the five components recommended in VR design of motor rehabilitation tasks (adaptability, feedback, task goals, interactivity and competition) (Zimmerli et al., 2013), it was expected that codes would be used from the following main taxonomy areas: goals and planning, feedback and monitoring, comparison of behavior, repetition and substitution, and antecedents, however coding was not restricted to these categories. The BCTs that are nested under these main taxonomies best align with the suggested VR design components identified by Zimmerli et al. (2013). Coders identified each BCT and gave the most appropriate corresponding code given definitions and descriptions in the BCTTv1. Prior to coding, both coders met to discuss standards for applying the BCTTv1 to VR task. This discussion was necessary to identify a systematic way in which to apply the BCTTv1 to the unique and dynamic nature of a VR task. After coding the first thirty-percent of the articles, both coders met and discussed areas of disagreement. Specifically, coders defined the use of antecedents as it applies to the virtual environment (VE) as this was the major area of disagreement. Following discussion, the coders agreed that the VR task would be given the code of "restructuring the physical environment" (taxonomy code: 12.1) if the VE had dynamic components that were added through the course of the VR task. Both coders also met after coding the remaining articles, again, to discuss areas of disagreement and establish a consensus regarding the BCTs being used in each VR intervention. Following this final meeting, a Cohen's Kappa statistic was calculated to demonstrate the level of agreement between coders.

\section{RESULTS}

\section{Study Selection and Characteristics}

Figure 2 illustrates the article selection process. The initial searches of PubMed, SPORTDiscus, and psycINFO yielded 708 articles. Of these 708 articles, 82 were duplicates. After removal of duplicate articles, 626 articles were screened through titles and abstracts. Through the screening process, 155 articles 


\begin{tabular}{|l|l|}
\hline Database & Search Algorithm \\
\hline SPORTDiscus & (virtual reality) AND (physical therapy or physiotherapy or rehabilitation) \\
& $\begin{array}{l}\text { AND (gait or ambulation or mobility or motor performance) } \\
\text { Refined by: English, Publication years 2007-2017 }\end{array}$ \\
\hline psycIFNO & $\begin{array}{l}\text { (virtual reality) AND (physical therapy or physiotherapy or rehabilitation) } \\
\text { AND (gait or ambulation or mobility or motor performance) } \\
\text { Refined by: English, Publication years 2007-2017 }\end{array}$ \\
\hline PubMed & $\begin{array}{l}\text { (virtual reality) AND (physical therapy or physiotherapy or rehabilitation) } \\
\text { AND (gait or ambulation or mobility or motor performance) } \\
\text { Refined by: English, Publication years 2007-2017 }\end{array}$ \\
\hline
\end{tabular}

FIGURE 1 | Database Search algorithm.

were excluded because they were a systematic review, metaanalysis, case study, feasibility study, study protocol, non-full text or non-research text. Additionally, 344 articles were excluded because they did not use neurologic populations, focused on the upper extremity, or did not investigate an upright mobility task. Following the screening process, 127 full text articles were reviewed for eligibility. Of these 127 articles, 95 were excluded due to the following reasons: did not use a VR task, did not directly measure the role of VR on motor performance, or were vestibular populations. The 32 remaining articles were included for review (Lamontagne et al., 2007; Yang et al., 2008; Kim et al., 2009; Mirelman et al., 2009, 2010, 2011; Brien and Sveistrup, 2011; Brütsch et al., 2011; Schuler et al., 2011; Salem et al., 2012; Park et al., 2013; Singh et al., 2013; Villiger et al., 2013, 2017; McEwen et al., 2014; Cho et al., 2015, 2016; Killane et al., 2015; Lee, 2015; Liao et al., 2015; Song and Park, 2015; Wall et al., 2015; Yom et al., 2015; Bang et al., 2016; In et al., 2016; Biffi et al., 2017; Bonney et al., 2017; Calabrò et al., 2017; Gandolfi et al., 2017; Pedreira da Fonseca et al., 2017; Peruzzi et al., 2017; van Gelder et al., 2017).

\section{Synthesized Findings Demographic Information}

As per the inclusion criteria for this review, all 32 studies had subjects with neurologic diagnoses (Table 1). Thirty of the articles were conducted in a single neurologic population including stroke $(n=15)$, Parkinson's disease $(n=4)$, spinal cord injury $(n=3)$, and cerebral palsy $(n=3)$, multiple sclerosis $(n=2)$, brain injury $(n=1)$, developmental delay $(n=1)$, and developmental coordination disorder $(n=1)$. The remaining two articles used mixed clinical populations; one had adults with stroke, spinal cord injury, or brain injury; the other used a pediatric population of cerebral palsy, transverse myelitis, multiple sclerosis or brain injury. Twenty-four articles used adult subjects. Eight articles used pediatric participants. Sample sizes ranged from 4 to 76 total participants. The majority of studies $(n=14)$ had sample sizes in the twenties. Regarding the study design, 21 studies were randomized control study designs. Six used a clinical group with repeated measures. Moreover, 17 studies conducted at least part of the study in a controlled research laboratory, 12 were in clinical settings, two were in a school system, and one was conducted at home via telerehabilitation.

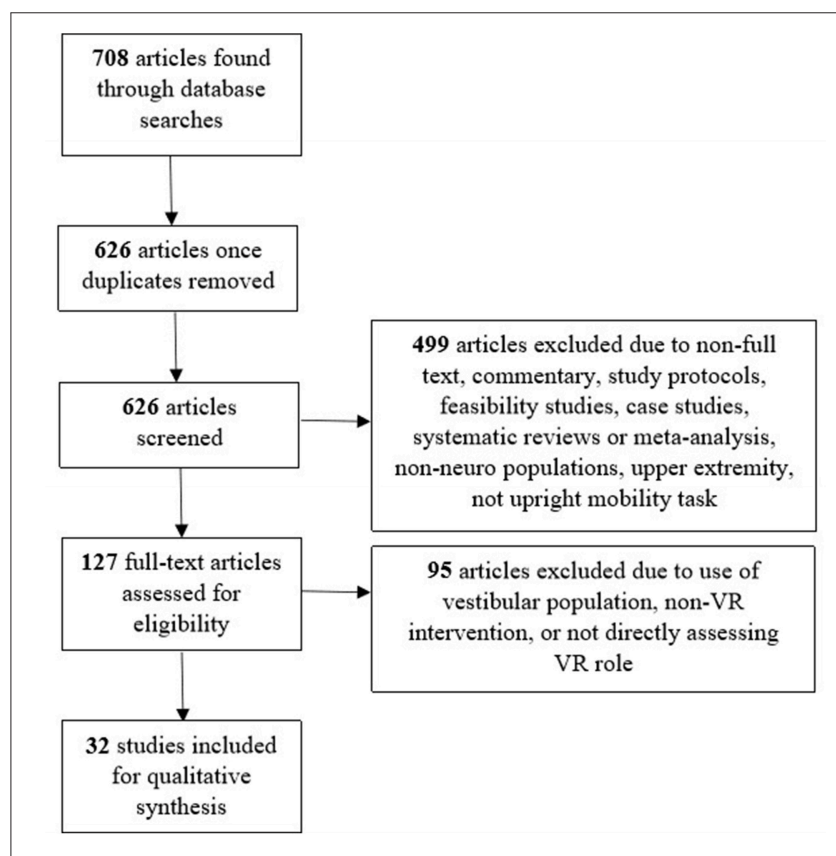

FIGURE 2 | Flowchart of systematic article search and inclusion for review.

\section{Virtual Reality Interventions}

Five of the 32 studies used an immersive form of VR. This was accomplished through either semi-cylindrical, multi-screen projection, or head mounted devices (HMDs). Twenty-seven of the 32 studies used a less-immersive VR intervention through use of 2D projection, IREX, RARS, Biorescue, YouRehab, WiiFit, or Xbox Kinect systems. A depiction of the VR systems and interventions of each study can be seen in Table 2 .

Eleven of the 32 studies used treadmill training in combination with their VR intervention. Three of these studies used robot-assisted gait training (RAGT), and eight used standard treadmill training (TT). One study used a balance platform which was integrated into their VR task. Two used the Rutgers ankle rehabilitation system (RARS). Three studies used the IREX VR system. Eight studies used commercial VR systems, Wii Fit $(n=7)$, or Kinect $(n=1)$, and one study used both the Nintendo Wii and Xbox Kinect. 
TABLE 1 | Summary of included articles investigating virtual reality interventions to improve motor performance in neurologic populations.

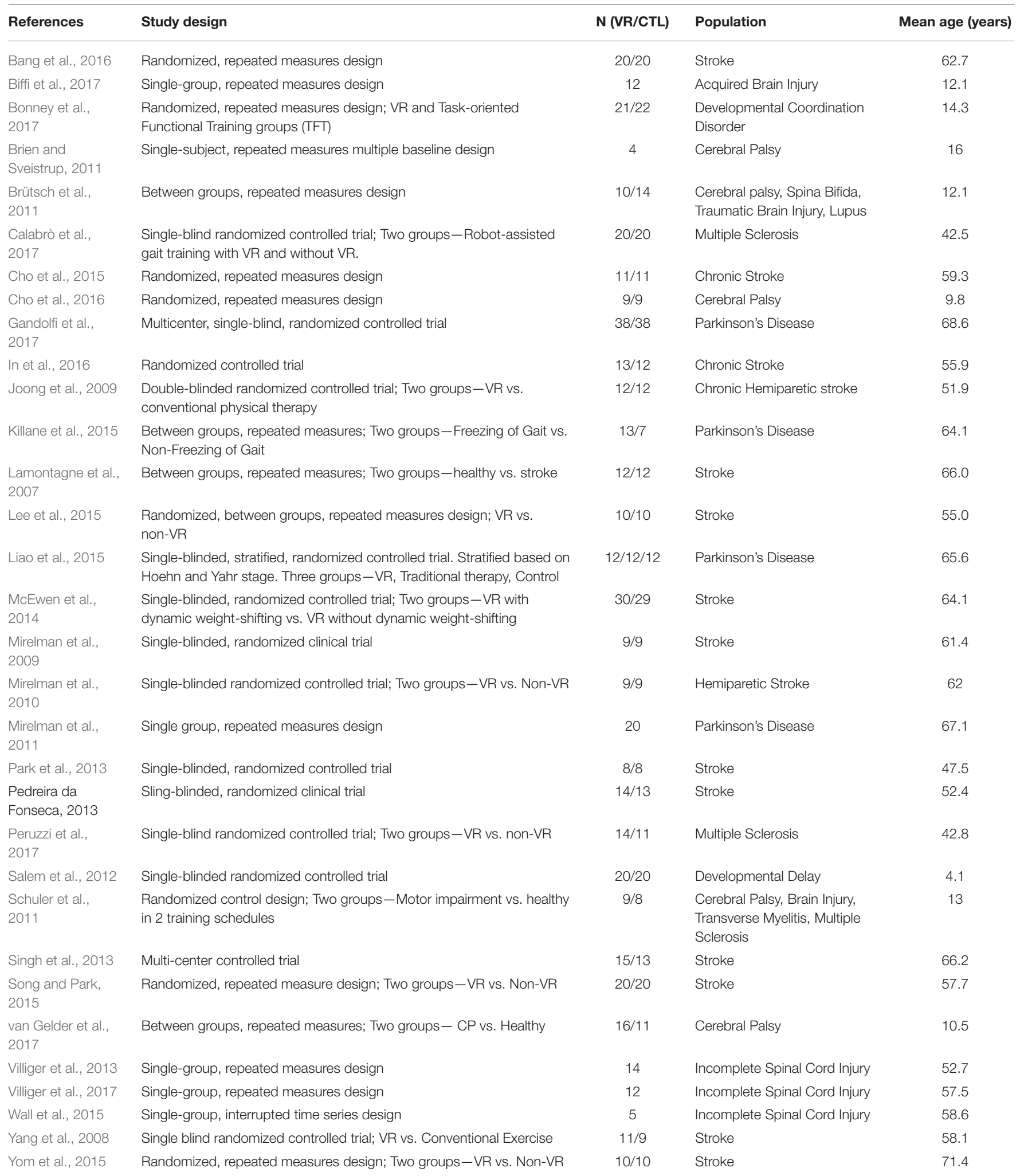


TABLE 2 | Summary of virtual reality interventions and findings of the included articles investigating virtual reality interventions on motor performance in neurologic populations.

\begin{tabular}{|c|c|c|c|}
\hline References & VR system & Virtual rehabilitation intervention & Findings \\
\hline Bang et al., 2016 & Nintendo Wii Fit & $\begin{array}{l}40 \text { min., } 3 \times / \text { week, } 8 \text { weeks. Performed yoga, muscular } \\
\text { strength exercise, aerobic exercise, and balancing } \\
\text { exercise for } 10 \mathrm{~min} \text {. each }\end{array}$ & $\begin{array}{l}\text { Significant improvement in balance for both groups } \\
\text { following exercise program; significant between group } \\
\text { differences. VR group demonstrated significant } \\
\text { differences for walking (affected limb stance and swing } \\
\text { phase, cadence) in post-testing. }\end{array}$ \\
\hline Biffi et al., 2017 & GRAIL system & $\begin{array}{l}\text { 10, } 30 \text { min. sessions over } 3 \text { weeks. Performed exercises } \\
\text { belonging to } 6 \text { groups; load transfer, monopodalic load, } \\
\text { walking, and endurance, dynamic balance, dynamic } \\
\text { balance joint range of motion, motor coordination. }\end{array}$ & $\begin{array}{l}\text { Significant improvement in gross motor abilities (standing } \\
\text { and walking), endurance, and autonomy in functional } \\
\text { activities. Significant decrease in the Gillette Gait Index } \\
\text { on impaired side, and improved symmetry. }\end{array}$ \\
\hline $\begin{array}{l}\text { Bonney et al., } \\
2017\end{array}$ & Nintendo Wii & $\begin{array}{l}45 \text { min., } 1 \times / \text { week, } 14 \text { weeks. Played } 8 \text { games per } \\
\text { session with progressive loading of training variables. }\end{array}$ & $\begin{array}{l}\text { Significant improvement in muscular strength, motor } \\
\text { proficiency, agility, and self-efficacy in both groups. No } \\
\text { significant difference between groups. }\end{array}$ \\
\hline $\begin{array}{l}\text { Brien and } \\
\text { Sveistrup, } 2011\end{array}$ & $\begin{array}{l}\text { Interactive Rehabilitation } \\
\text { and Exercise system (IREX) }\end{array}$ & $\begin{array}{l}\text { 2, } 45 \text { min. sessions, } 30 \text { min. rest, } 5 \text { consecutive days. } \\
\text { Performed } 3 \text { consecutive sets of } 5 \text { IREX applications }\end{array}$ & Significant improvement in balance and gait. \\
\hline
\end{tabular}

each session. Applications lasted 2 min., with $10 \mathrm{~s}$. rest

interval. Games consisted of soccer, snowboard, sharkbait, zebra crossing, and gravball.

$\begin{array}{ll}\begin{array}{l}\text { Bütsch et al., } \\ 2011\end{array} & \begin{array}{l}\text { Lokomat with 42-inch } \\ \text { screen }\end{array} \\ \begin{array}{l}\text { Calabrò et al., } \\ 2017\end{array} & \text { Lokomat-Pro } \\ \text { Cho et al., 2015. } & \begin{array}{l}\text { Treadmill with VR } \\ \text { environment projected onto } \\ \text { a } 1,800 \times 1,900 \text { mm screen }\end{array}\end{array}$

4 randomly assigned conditions; 3 (30sec) validation trials, 7 min. treatment trials; VR trials consisted of soccer and navigation.

40 min. of RAGT + VR, 5x/week, 8 weeks with interactive avatar in VE. Subjects required to pass obstacles or catch objects appearing on a trail.

30 min., $5 \times /$ week. Subjects walked on the treadmill and viewed the VE while performing cognitive tasks. VE included real community environment projections (crosswalk, garden, etc.). Cognitive tasks included memorization, math, and verbal tasks.

Cho et al., $2016 \quad$ Treadmill with 42-inch TV projection; Nintendo Wii jogging program.

Gandolfi et al., 2017 Nintendo Wii; Web-camera attached to computer to provide telerehabilitation via skype.

In et al., 2016 Virtual Reality Reflection Therapy (VRRT)

Joong et al., 2009 Interactive Rehabilitation and Exercise System (IREX).

Killane et al., 2015 VR maze game designed by DFKI, Germany. Fifty five inch screen, 1 meter in front of participant. Wii to produce navigation in the VE.

Lamontagne et al., Kaiser Optics ProView ${ }^{T M}$ 2007 XL50 (HMD). VE powered by CAREN-2 system (Motek). hallway. 1) continuously changing optic flow sinusoidally

Lee et al., $2015 \quad$ BioRescue platform.
Single collection; 2 conditions while walking in a virtual from $0-2 x$ participant's comfortable walking speed 2) optic flow changes at discrete, constant speeds $0.25-2 \times$ participant's comfortable walking speed.

30 min., $3 \times /$ week, 8 weeks. Walked on treadmill with jogging program

50 min., 3x/week, 7 weeks. Exercised on Wii only during ON state performing 10 exergames; table tilt, penguin slide, balance bubble, ski slalom, skateboarding, perfect 10, tilt city, snowball fight, rhythm parade, and birds-eye bulls-eye.

Both groups performed 30 min. of convention physical therapy. Then, VR group performed 30 min., $5 \times$ /week, 4 weeks of VRRT intervention.

30 min., 4x/week, 3 weeks in addition to 40 minutes of conventional physical therapy. Intervention targeted a weight-shifting, balance and stepping tasks

20 min., 8 session, 2 weeks. VR maze which participates navigated while stepping-in-place. Time constraints and cognitive dual-tasks were added.

\subsection{5-2x participant's comfortable walking speed.}

$45 \mathrm{~min} ., 3 \times /$ week, 6 weeks. City
and bubble activities were used.
Significant main effect for training condition. Improved effectiveness in initiating active participation in VR conditions.

Small effect size and non-significant differences between groups favoring RAGT+VR for Berg Balance Scale and TUG.

Improvement in walking function under both single and dual task conditions, as well as for VR and control groups. Greater improvements in function for the VR group under dual task condition compared to control.

Gait and balance improved following training for the VR group than control.

Significant difference between-group on balance for VR group, and significant time $X$ group interaction for gait improvements in clinic group. Improvements in all outcomes measures for both groups except frequency of falls.

Significant improvements in static and dynamic standing balance, and gait speed in VR group compared to control.

Significant improvement in dynamic standing balance, gait speed, cadence, step time, step length and stride length in VR group. Correlation between improved dynamic balance and gait velocity.

Significant improvement in dual-tasking, stepping time, stepping rhythmicity. Improvement in FOG episodes. Improvements great in the FOG group vs. Non-FOG.

Overall, demonstrated negative correlation between gait speed and optic flow speed. This relationship was weaker in stroke subjects than healthy subjects.

Significant improvement in dynamic standing balance following intervention in VR group and compared to control. 
TABLE 2 | Continued

\begin{tabular}{lll}
\hline References & VR System & Virtual Rehabilitation Intervention \\
\hline Liao et al., 2015 & Wii Fit & $\begin{array}{l}45 \text { min., } 2 \times / \text { week, } 6 \text { weeks. Interactive avatar, yoga, } \\
\text { strength, and balance exercises. 10, 15, and 20 min., } \\
\text { respectively. }\end{array}$ \\
McEwen et al., & $\begin{array}{l}\text { Interactive Rehabilitation } \\
\text { and Exercise System (IREX) }\end{array}$ & $\begin{array}{l}\text { 10-12, 20 min. sessions. Standing VR games which } \\
\text { dynamic weight-shift such as soccer and snowboarding } \\
\text { were used. }\end{array}$
\end{tabular}

Mirelman et al., Rutgers Ankle Rehabilitation 60 min., 3x/week, 4 weeks. Seated ankle dorsiflexion, $2009 \quad$ System (RARS)

Mirelman et al., $\quad$ Rutgers Ankle Rehabilitation 60 min., 3x/week, 4 weeks. Navigated a plane or boat 2010 System (RARS)

Mirelman et al., Outdoor virtual scene 2011

Park et al., 2013 Head-mount device (HMD)

Pedreira da

Fonseca et al., 2017

Peruzzi et al., 2017

Salem et al., 2012 Nintendo Wii Sports and Nintendo Wii Fit

VE generated with WorldViz and projected on a 27 -inch screen.

Nintendo Wii

$3 \times /$ week., 6 weeks. Process multiple stimuli and make decisions about obstacle negotiation in 2 planes, with distracters such as changing light and moving objects, while walking on a treadmill.

Both groups performed 60 min., $5 \times$ /week, 4 weeks of conventional physical therapy. VR group performed additional $30 \mathrm{~min}$. $3 \times /$ week, 4 weeks of VR intervention. Three-stage program: (1) supine trunk stability and pelvic tilt, (2) sitting trunk stability and pelvic tilting, and selective movements between each, (3) lower extremity muscle strengthening, standing trunk stability.

$2 \times /$ week for 20 sessions of conventional physical therapy. VR group received $15 \mathrm{~min}$ of conventional physical therapy plus $45 \mathrm{~min}$. VR intervention. Tennis, hula hoop, soccer and boxing were used.

45 min., $3 \times /$ week, 6 weeks. Virtual tree-line trail, passing objects appearing on the trail.

30 min., $2 \times$ /week, 10 weeks. VR training used balance, strength and walking games; lunges, single leg stance, soccer, penguin slide, tightrope, running, hula hoop, etc.

Schuler et al., Lokomat 2011

Singh et al., 2013 Nintendo Wii Fit and Xbox 360 Kinect

Song and Park, Xbox Kinect

2015

van Gelder et al., Gait Real-time Analysis 2017 Interactive Lab (GRAIL).

Villiger et al., 2013 YouRehab, YouKicker system

$14 \mathrm{~min} . /$ session. Two types of VR games; soccer game with virtual opponents and a landscape with objects to collect.

30 min., $2 \times /$ week, 6 weeks. Performed Balance Bubble on Wii Fit and Rally Ball on Kinect. Participants who mastered Rally Ball progressed to Reflex Ridge.

30 min., $5 \times /$ week, 8 weeks. Kinect sports, sports season 2, adventure and gunstringer were used.

2 trials/2 min. each. Visual feedback regarding knee and hip extension by presenting a bar plot with vertically moving ball and a target to visualize the goal knee/hip extension.

45 min., 4-5x/week, 4 weeks. Footbag, Hamster Splash, Stark Kick, and Planet Drive were used. plantar flexion, inversion, eversion and combined motions were performed to navigate a boat or train through a VE with movable targets. through the VE which contained a series of targets using only dorsiflexion, plantar flexion, inversion and eversion, or a combination of these movements.

\section{Findings}

Greater improvement in obstacle crossing velocity, crossing stride length, dynamic balance, SOT, TUG, FES-I and PDQ39 in VR group than control. VR group also demonstrated significant improvement in movement velocity of limits-of-stability test than TE training.

Both groups improved dynamic balance and gait speed at the minimal clinical important difference for each outcome measure post-training. Effect sizes favored experimental group.

Improvements in gait speed and distanced walked in VR group. Significant improvements in distance and steps walked in community in VR group which were maintained 3-months post-intervention.

VR group demonstrated significantly larger increase in ankle power generation in push-off, greater change in ankle ROM post-training, and significant differences in knee ROM on affected side during stance and swing. No significant differences in kinematics or kinetics at hip.

Significant improvement in gait speed during usual walking, dual tasks, and over-ground obstacle negotiation. Dual-task variability and Trail Making Test times improved. Retention effects noted 1 month later.

Significant improvement in gait parameters (except cadence) in VR group post-intervention, and at 1-month retention. No significant change in control group. Significant improvement in stride length in VR group compared to control.

Improvement in dynamic balance and a reduction in falls in both VR and control groups. Significant reduction of falls demonstrated in VR group. Significant change in dynamic balance in control group.

Significant improvements in walking endurance, speed, cadence, stride length, and lower extremity ROM in both groups. VR group significantly improved balance. VR group improved significantly more than control group in hip ROM and hip power at terminal stance in post-training.

Significant improvement in single-limb balance and grip strength in VR group vs. control. Demonstrated trend toward greater improvement in dynamic balance, gait parameters and gross motor function in VR group vs. control.
EMG activity significantly higher in both groups during tasks with VR than normal walking conditions.

Significant improvement in the VR group for dynamic balance, however non-significant between-group findings.

Improvement in balance and weight bearing in hemiparetic side in both groups.

All children, except one, demonstrated improvement in hip and/or knee extension during gait in response to real-time feedback. Peak hip extension and peak knee extension significantly improved.

Significant improvements in gait parameters, balance and strength of lower limbs following treatment and maintained 12-16 weeks after training. 
TABLE 2 | Continued

\begin{tabular}{|c|c|c|c|}
\hline References & VR System & Virtual Rehabilitation Intervention & Findings \\
\hline Villiger et al., 2017 & $\begin{array}{l}\text { YouRehab, YouKicker } \\
\text { system }\end{array}$ & $\begin{array}{l}\text { 30-45 min., 16-20 session, } 4 \text { weeks. Footbag, Planet } \\
\text { Drive, Star Kick, Hamster Splash were used. }\end{array}$ & $\begin{array}{l}\text { Significant improvements in lower limb strength, balance } \\
\text { and functional mobility. Significant improvement in } \\
\text { functional mobility maintained at 2-3-month retention. }\end{array}$ \\
\hline Wall et al., 2015 & Nintendo ${ }^{\mathrm{TM}}$ Wii Fit & $\begin{array}{l}60 \text { min., } 2 \times / \text { week, } 7 \text { weeks. Performed multiple games } \\
\text { from Wii Fit to improve weight shifting, stability, balance } \\
\text { and coordination. }\end{array}$ & $\begin{array}{l}\text { Significant improvement in gait speed and dynamic } \\
\text { balance following training, which were maintained } \\
\text { 4-weeks post-training. }\end{array}$ \\
\hline Yang et al., 2008 & $\begin{array}{l}\text { Visual screen, 3D } \\
\text { acceleration graphics and } \\
\text { 3D audio }\end{array}$ & $\begin{array}{l}20 \text { min., 3x/week, } 3 \text { weeks. VE simulated a community in } \\
\text { Taipei and was integrated to speed and incline changes } \\
\text { of treadmill; scenarios included lane walking, street } \\
\text { crossing, obstacle striding and park stroll. }\end{array}$ & $\begin{array}{l}\text { VR group significantly improved walking speed, } \\
\text { community walking times and WAQ score post-training } \\
\text { and in } 1 \text { month retention. VR group significantly } \\
\text { improved ABC which was not maintained at retention. } \\
\text { VR group improved significantly more than controls. }\end{array}$ \\
\hline Yom et al., 2015 & $\begin{array}{l}\text { Virtual reality-based ankle } \\
\text { exercise (VRAE) using VRAE } \\
\text { exercise program, computer } \\
\text { and projector. }\end{array}$ & 30 min., $5 \times$ /week, 6 weeks. & $\begin{array}{l}\text { Significant improvement in dynamic balance and muscle } \\
\text { tone following VR intervention and compared to control } \\
\text { group. }\end{array}$ \\
\hline
\end{tabular}

All 32 studies reported improvements in the primary motor outcomes specific to each intervention with the use of VR. None of the studies compared the efficacy of immersive and nonimmersive VR technologies. Additionally, none of the studies investigated the specific components of their intervention (i.e., adaptability, feedback, competition, etc.) to analyze the true effectiveness of each component.

\section{Behavior Change Technique Coding}

The number of studies that included at least one of the recommended BCT components are present in Figure 3. A breakdown of which components were included in each study is present in Table 3. There was substantial agreement (Cohen's Kappa $=0.714)$ for the initial thirty-percent of articles coded, improving to almost perfect agreement (Cohen's Kappa $=0.961$ ) for the final seventy-percent of articles coded following the consensus meeting between the two coders. Eighteen studies (56.3\%) used graded tasks (taxonomy code: 8.7 ) to adapt the VR intervention to optimally challenge the participants. For example, Villiger et al. (2013) graded task difficulty by adjusting the speed of the task or adding obstacles, which participants were instructed to avoid, in their VE. Fifteen studies (46.9\%) provided explicit feedback to participants, six used feedback on performance of the behavior (taxonomy code: 2.2), and nine used feedback of outcomes on behavior (taxonomy code: 2.7 ). van Gelder et al. (2017) gave subjects feedback on performance of their target behavior through visual feedback provided via a bar plot and vertical ball which moved according to real-time joint angles in reference to a visual target. Liao et al. (2015) provided feedback on outcomes of behavior by providing subjects with their total score following conclusion of the VR task. The interactivity between the subjects' real-world movements and movements of the avatar in the VR is an example of biofeedback (taxonomy code: 2.6) which was used in nineteen $(59.4 \%$ ) of studies. One (3.1\%) set explicit goals related to behavior outcome (taxonomy code:1.3) by explaining the goals of the VR training prior to beginning the protocol (Bonney et al., 2017). The option for adding competition to the VR task was not included in any study in this review. VR interventions which had a dynamic component to their VE were coded as "restructuring the physical environment" (taxonomy code: 12.1). Yang et al. (2008) accomplished this through by creating a VE with three-dimensional graphics and auditory outputs, which changed with the speed and incline of the treadmill used in the intervention. Five studies (15.6\%) were coded as restructuring the physical environment.

\section{DISCUSSION}

\section{Summary of Main Findings}

The purpose of this study was to systematically review the existing VR literature aimed at improving motor performance in neurologic populations and analyze the underlying BCTs by coding each intervention using the BCTTv1. Studies were included if they directly investigated the role of VR in improving gross motor performance in neurologic populations. Only the VR component of the intervention was considered during the BCT coding. Thirty-two studies were included in total.

Overall, it is clear that VR interventions for motor rehabilitation of neurologic populations is in its infancy. There is limited diversity in patient populations, most studies use adult participants, and many of the studies were performed in controlled environments. Post-stroke populations were the most commonly studied, used in $46.9 \%$ of the studies included in this review. Also, most studies used a non-immersive form of VR intervention. Moreover, none of the articles investigated the efficacy of individual components of their VR intervention to better understand the significance of the motor performance outcomes. All studies reported improvements in motor outcomes, however it is difficult to point to the most effective component if systematic investigation into the specific parts of a VR intervention is not performed, especially when trying to analyze the most effective active ingredients, or BCTs, of that intervention.

Improvements in technology have also increased the usage of telerehabilitation to enhance home-based physical rehabilitation 


\section{BCT usage for the recommended components of VR interventions}

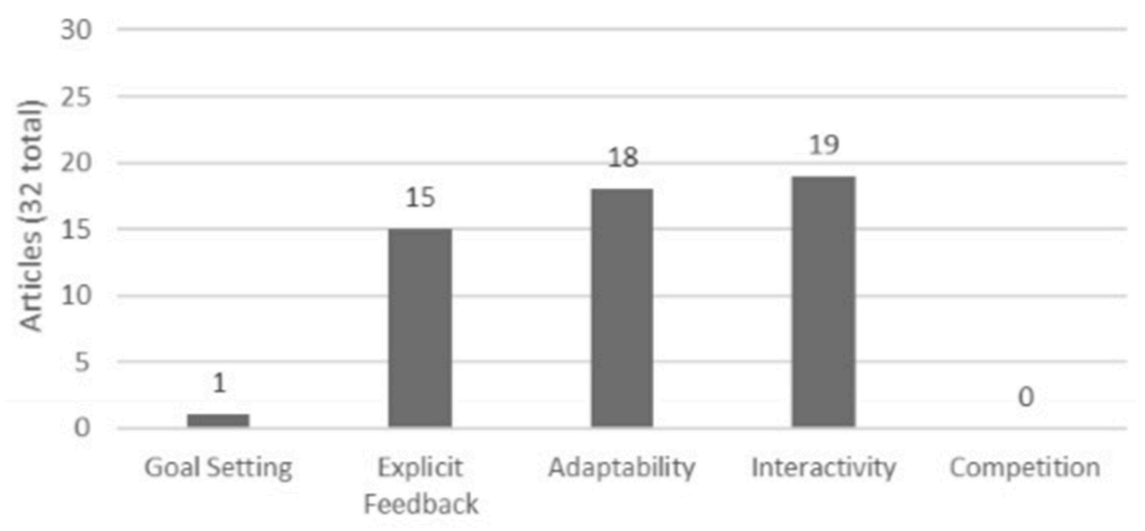

FIGURE 3 | Summary of BCT usages in respect to the 5 recommended components of VR interventions across all 32 articles.

(Latifi, 2008). There has been emerging investigation of the role of VR in telerehabilitation of the upper extremity as well as balance (Popescu et al., 2000; Golomb et al., 2010; Llorens et al., 2015). However, only one study investigated the use of VR in telerehabilitation of upright mobility (Gandolfi et al., 2017). Further investigation into the feasibility, efficacy and safety of VR interventions included in telerehabilitation protocols is needed.

Goal setting, explicit feedback, adaptability, interactivity, and competition have been identified as components of a successful VR task (Zimmerli et al., 2013). Given these recommended components, it was expected that codes would be used from the following main taxonomy areas: goals and planning, feedback and monitoring, comparison of behavior, and repetition and substitution. Goal setting and planning includes BCTs related to setting explicit task goals. These components were not readily used, as they were only incorporated in 1 of 32 studies $(3.1 \%)$ of the articles in this review. Feedback and monitoring include BCTs related to providing feedback, monitoring behavior, and biofeedback. This section of the taxonomy applies to both the explicit feedback and interactivity components recommended for VR tasks, and at least one BCT from this area of the taxonomy was used in most of the studies included in this review (21 out of 32 studies; 65.6\%). Fifteen studies utilized explicit feedback (46.9\%) with studies providing either feedback on performance or feedback on outcomes of behavior. Biofeedback is defined in the BCTTv1 as a method of providing feedback about the body using an external monitoring device. Through use of interactive VR technologies, users can readily receive feedback from the VR. Therefore, interactivity of a VR task can be coded as using biofeedback, which was incorporated in 19 of 32 studies (59.4\%) included in this review. Finally, the repetition and substitution section of the taxonomy includes "graded task," which is used to set an easily attainable task that can gradually be increased in difficulty to achieve the desired outcome. This also speaks to the adaptability of a VR task. Of the recommended components of $\mathrm{VR}$, adaptability was the most commonly adopted component, being incorporated into 18 out of 32 studies (56.3\%) included in this review. These results illustrate the state of BCTs in VR tasks for neurorehabilitation. BCTs related to interactivity, feedback and adaptability of an intervention are represented well, however BCTs for explicit task goals and competition are lacking in the interventions included in this review. As suggested by Zimmerli et al. (2013), to create well-rounded VR interventions aimed to improve motor performance it is imperative to include multiple components into the VR task design. Therefore, interventions that fail to include aspects of goal setting, for example, may lose an important active ingredient to maximize the effectiveness of the VR intervention on neurorehabilitation and motor outcomes.

BCTs are often not deliberately stated in descriptions of behavioral interventions (Michie et al., 2009), and this holds true in the VR literature for motor rehabilitation. Thus, some aspects of the VR interventions that could be implied, but did not explicitly state the target behavior, could not be coded. Moreover, none of the included articles investigated individual components of their VR intervention. For this reason, it is difficult to identify the BCTs which provide the optimal augmentation of the VR intervention. However, interventions using graded tasks (taxonomy code: 8.7) and biofeedback (taxonomy code: 2.6) attributed the improved motor performance from the intervention to these components of the VR task. Graded tasks were used in 18 studies (56.3\%), and biofeedback in 19 studies (59.4\%). Twelve articles (37.5\%) had VR tasks that used adaptability and interactivity together. A graded task is defined in the BCTTv1 as an intervention created to be initially easy to perform with increasing difficulty until the target behavior is accomplished. This speaks to the adaptability of the intervention. Biofeedback provides feedback about the state of the body using external monitoring. This suggests a level of interactivity in the VR task. This review suggests that these components improve patient engagement and motivation in the motor task (Schuler et al., 2011; Zimmerli et al., 2013) and should be considered for inclusion in future research. 


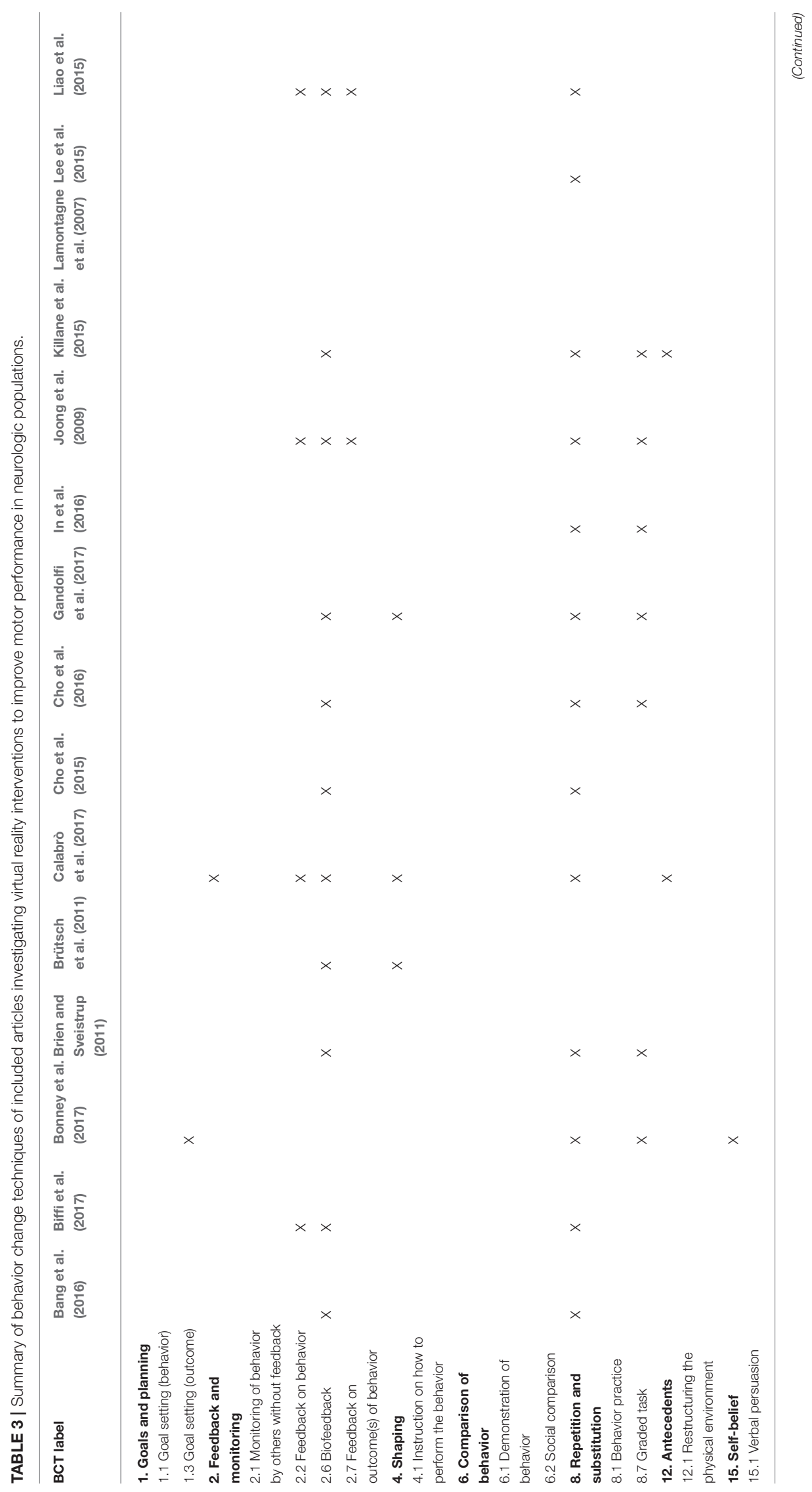


Felsberg et al.

Virtual Reality and Neurorehabilitation

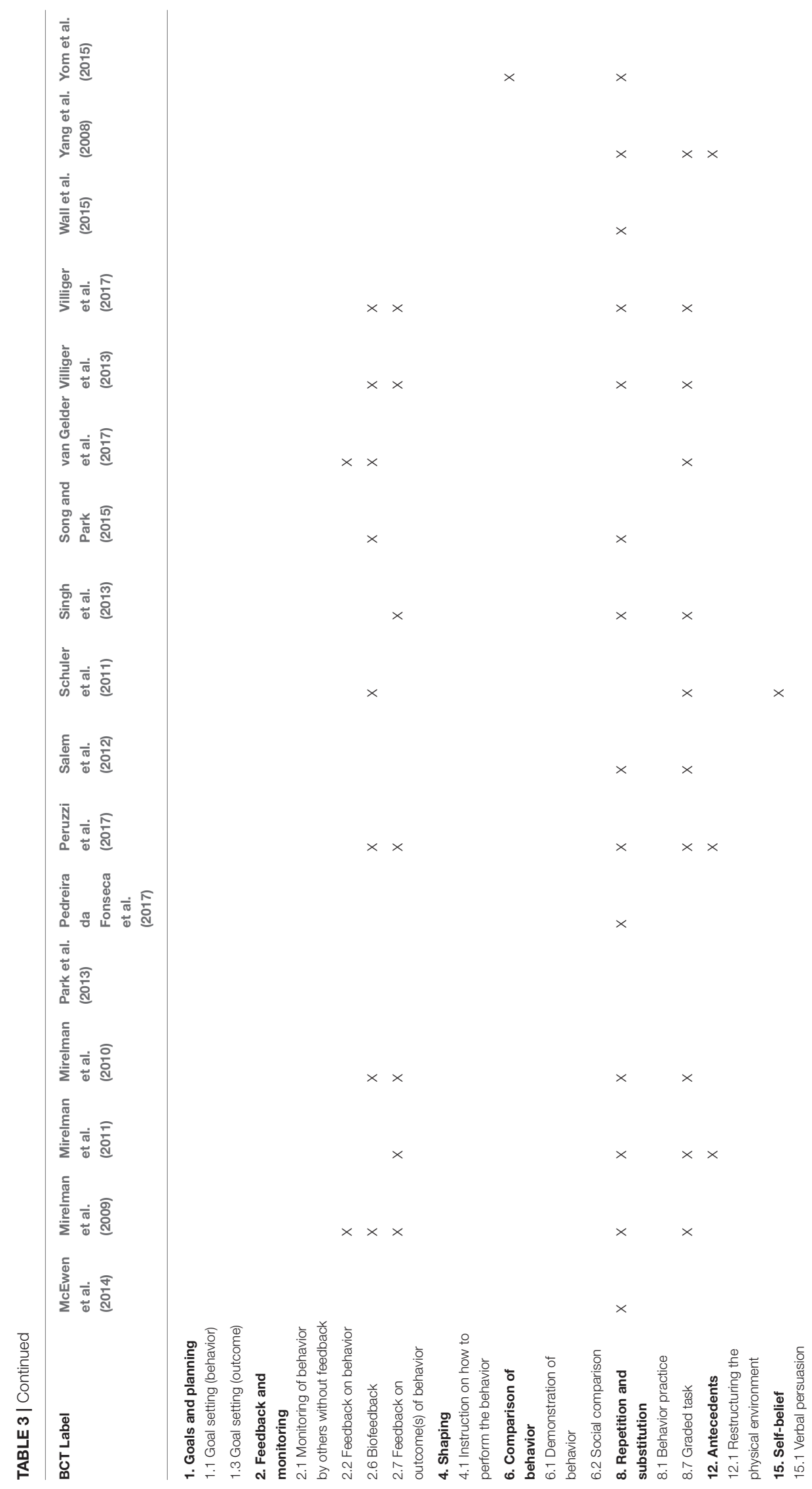

Frontiers in Psychology | www.frontiersin.org

11

May 2019 | Volume 10 | Article 979 
As previously stated, only one article investigated the efficacy of individual components of their VR task. Zimmerli et al. found that adaptability leads to improvements in motor outcomes by allowing the VR task to be adapted to meet the individual needs of the user, and this produces the ideal psychophysiological state in which a participant is optimally stimulated, both mentally and physically (Zimmerli et al., 2013). Interactivity helps improve task-engagement, as well as optimizing rehabilitation potential and motor outcomes. Conversely, components of feedback frequency and goal setting were found to not produce a significant improvement in the motor performance of subjects with neurologic disorders.

In terms of neurologic rehabilitation, the goal of including tasks is to change behavior to improve motor outcomes. Behavior change is complex, requiring a combination of multiple components to produce the desired outcome (Michie et al., 2013). In this way, it is important to identify the BCTs currently used successfully in VR literature, as well as the shortcomings. In this review, coders looked for BCTs related to the specific recommended components of a VR intervention, as well as identify BCTs present in the VR task which were outside of these recommendations. In terms of the recommended VR components and their associated BCTs, feedback, adaptability and interactivity are the most widely used in the thirty-two articles reviewed. However, the remaining two components, goal setting and competition, were not incorporated well into the VR tasks included in this review. Outside the 5 recommended components, the most used BCT overall was behavioral practice (taxonomy code: 8.1 ); $84.4 \%$ of the studies created their VR intervention to rehearse the performance of the desired behavior which is important with regard to motor rehabilitation. Changing the environment to facilitate the desired behavior change is deemed restructuring the physical environment (12.1), this BCT was used in $15.6 \%$ of the studies. These studies created a dynamic component within their VR which was not present at the beginning of the task, restructuring the VE to influence the wanted behavior change.

From this review, it was observed that some BCTs are being integrated into VR tasks to meets the needs of the user and create meaningful changes in motor performance. These include feedback (behavior and outcomes), graded tasks, biofeedback and behavioral practice, and have been shown to change physical activity behavior (Duff et al., 2017; Samdal et al., 2017). Conversely, from the BCT literature, it has been shown that goal setting and self-monitoring is effective for creating behavior change in general (Michie et al., 2009). Self-monitoring of behavior was not used in any of the articles in this review, and goal setting was only incorporated into $3.1 \%$ of the studies in this review.

This review provides important information regarding the use of BCTs in the design of VR interventions to improve motor outcomes in neurologic clinic populations. Specifically, this review investigated the components of each VR intervention, itself, rather than the complete intervention protocol. Some BCTs are being incorporated well while others are not. However, more comprehensive VR tasks designed with deliberate integration and testing of BCTs are needed. This will allow for better identification of the true active ingredients promoting change within a successful VR task, which BCTs are best for VR interventions in motor rehabilitation and improve replicability of clinically meaningful VR interventions. Addressing these gaps will allow for more tailored treatments to be developed in the physical rehabilitation domain, similar to those currently being implemented in the cognitive rehabilitation domain (Freeman, 2008; Parsons and Rizzo, 2008; Li et al., 2011; Wang and Reid, 2011; Gonçalves et al., 2012; Opriş et al., 2012; Kandalaft et al., 2013). This will help spring-forward this line of research to more rapidly catch up with its cognitive rehabilitation counterparts to more fully take advantage of VR therapy across multiple domains.

\section{Limitations}

There are two limitations of note in this review. First, there is not an established way to apply the BCTTv1 to VR interventions. Other articles performing BCT coding using the BCTTv1 have established a clear framework for applying BCT codes to various technological interventions. However, these are mostly regarding mobile phone applications (Direito et al., 2014, 2016; Lyons et al., 2014; Middelweerd et al., 2014; Yang et al., 2015). Additionally, while some BCTs could be implied, if the text did not explicitly state or link the target behavior to the target population, it could not be coded.

\section{CONCLUSION}

The purpose of this study was to investigate the ways in which VR was being used in motor rehabilitation of neurologic populations, and systematically code these interventions using the BCTTv1 to analyze the active ingredients of each VR task. The literature suggests that VR is a useful modality to design and implement effective rehabilitation interventions. However, the state of BCTs in VR is mixed. The literature suggests that graded tasks (adaptability) and biofeedback (interactivity) are two of the most consistent elements of a VR intervention for motor rehabilitation. BCTs are not often deliberately reported in the literature, and therefore cannot be coded. Moreover, studies did not investigate the specific components of their VR tasks making it difficult to link BCTs to significant components of VR interventions.

Further research into the specific components of VR interventions along with purposeful implementation and reporting of BCTs will help improve understanding of the efficacy of VR as a motor rehabilitation tool. Incorporation of BCTS into VR interventions could create the ideal intervention to enhance motor outcomes. Future research could benefit from incorporating BCTs, and what is currently known about BCTs from the physical activity literature, into the design process of VR interventions to produce optimal rehabilitation potential. However, it is acknowledged that the BCT framework is rarely used in physical rehabilitation intervention, and questions remain about its applicability to help enhance current practice. Nevertheless, we believe the inclusion of BCTs a priori could help our field systematically 
understand the extent to which VR could add value to current clinical approaches.

\section{AUTHOR CONTRIBUTIONS}

All authors contributed to the concept and design of the systematic review. DF performed the literature search. DF and JM performed the coding of all included articles according to the BCTTv1. DF drafted the initial manuscript. All authors contributed to manuscript revision and approved the manuscript for submission.

\section{REFERENCES}

Abraham, C., and Miche, S. (2008). A taxonomy of behavior change techniques used in interventions. Health Psychol. 27, 379-387. doi: 10.1037/0278-6133.27.3.379

Aida, J., Chau, B., and Dunn, J. (2018). Immersive virtual reality in traumatic brain injury rehabilitation: a literature review. NeuroRehabilitation 42, 441-448. doi: 10.3233/NRE-172361

Bang, Y., Son, K., and Kim, H. (2016). Effects of virtual reality training using nintendo wii and treadmill walking exercise on balance and walking for stroke patients. J. Phys. Ther. Sci. 28, 3112-3115. doi: 10.1589/jpts.28.3112

Biffi, E., Beretta, E., Cessareo, A., Maghini, C., Turconi, A., Reni, G., et al. (2017). An immersive virtual reality platform to enhance walking ability of children with acquired brain injuries. Methods Inform. Med. 56, 119-126. doi: 10.3414/ME16-02-0020

Bird, E., Baker, G., Mutrie, N., Ogilvie, D., Sahlqvist, S., and Powell, J. (2013). Behavior change techniques used to promote walking and cycling: a systematic review. Health Psychol. 32, 829-838. doi: 10.1037/a0032078

Bonney, E., Ferguson, G., and Smits-Engelsman, B. (2017). The efficacy of two activity-based interventions in adolescents with developmental coordination disorder. Res. Dev. Disabil. 71, 223-236. doi: 10.1016/j.ridd.2017.10.013

Brien, M., and Sveistrup, H. (2011). An intensive virtual reality program improves functional balance and mobility of adolescents with cerebral palsy. Pediatr. Phys. Ther. 23, 258-266. doi: 10.1097/PEP.0b013e318227ca0f

Brütsch, K., Koenig, A., Zimmerli, L., Mérillat-Koeneke, S., Riener, R., Jäncke, L., et al. (2011). Virtual reality for enhancement of robot-assisted gait training in children with central gait disorders. J. Rehabil. Med. 43, 493-499. doi: 10.2340/16501977-0802

Calabró, R., Russo, M., Naro, A., De Luca, R., Leo, A., Tomasello, P., et al. (2017). Robotic gait training in multiple sclerosis rehabilitation: can virtual reality make the difference? Findings from a Randomized Controlled Trial. Neurol. Sci. 377, 25-30. doi: 10.1016/j.jns.2017.03.047

Casuso-Holgado, M., Martín-Valero, R., Carazo, A., Medrano-Sánchez, E., Cortés-Vega, M., and Montero-Bancalero, F. (2018). Effectiveness of virtual reality training for balance and gait rehabilitation in people with multiple sclerosis: a systematic review and meta-analysis. Clin. Rehabil. 32, 1220-1234. doi: 10.1177/0269215518768084

Cho, C., Hwang, W., Hwang, S., and Chung, Y. (2016). Treadmill training with virtual reality improves gait, balance, and muscle strength in children with cerebral palsy. Tohoku J. Exp. Med. 238, 213-218. doi: 10.1620/tjem.238.213

Cho, K., Kim, M., Lee, H., and Lee, W. (2015). Virtual reality training with cognitive load improves walking function in chronic stroke patients. Tohoku J. Exp. Med. 236, 273-280. doi: 10.1620/tjem.236.273

Conroy, D., Yang, C., and Maher, J. (2014). Behavior change techniques in topranked mobile apps for physical activity. Am. J. Prev. Med. 46, 649-652. doi: 10.1016/j.amepre.2014.01.010

Darekar, A., Lamontagne, A., and Fung, J. (2015). Dynamic clearance measure to evaluate locomotor and perceptuo-motor strategies used for obstacle circumvention in a virtual environment. Hum. Mov. Sci. 40, 359-371. doi: 10.1016/j.humov.2015.01.010

Direito, A., Carraça, E., Rawstorn, J., Whittaker, R., and Maddison, R. (2016). MHealth technologies to influence physical activity and sedentary

\section{FUNDING}

This work was supported by partial funding from the University of North Carolina at Greensboro's Open Access Publishing Support Fund.

\section{ACKNOWLEDGMENTS}

The authors would like to thank Chih-Hsiang (Jason) Yang, Ph.D., postdoctoral research associate at University of Southern California, for his assistance with the calculation of Cohen's Kappa.

behaviors: behavior change techniques, systematic review and metaanalysis of randomized controlled trials. Ann. Behav. Med. 51, 226-239. doi: 10.1007/s12160-016-9846-0

Direito, A., Dale, L., Shields, E., Dobson, R., Whittaker, R., and Maddison, R. (2014). Do physical activity and dietary smartphone applications incorporate evidence-based behaviour change techniques? BMC Public Health 14:646. doi: 10.1186/1471-2458-14-646

Duff, O., Walsh, D., Furlong, R., O’Connor, N., Moran, K., and Woods, C. (2017). Behavior change techniques in physical activity EHealth interventions for people with cardiovascular disease: systematic review. J. Med. Internet Res. 19:e281. doi: 10.2196/jmir.7782

Ellis, T., Boudreau, J., DeAngelis, T., Brown, L., Cavanaugh, J., Earhart, G., et al. (2013). Barriers to exercise in people with Parkinson disease. Phys. Ther. 93, 628-636. doi: $10.2522 /$ ptj.20120279

Freeman, D. (2008). Studying and treating schizophrenia using virtual reality: a new paradigm. Schizophr. Bull. 34, 605-610. doi: 10.1093/schbul/sbn020

Gandolfi, M., Geroin, C., Dimitrova, E., Boldrini, P., Waldner, A., Bonadiman, S., et al. (2017). Virtual reality telerehabilitation for postural instability in Parkinson's disease: a multicenter, single-blind, randomized, controlled trial. Biomed. Res. Int. 2017:7962826. doi: 10.1155/2017/7962826

Golomb, M., McDonald, B., Warden, S., Yonkman, J., Saykin, A., Shirley, B., et al. (2010). In-home virtual reality videogame telerehabilitation in adolescents with hemiplegic cerebral palsy. Arch. Phys. Med. Rehabil. 91, 1-8.e1. doi: 10.1016/j.apmr.2009.08.153

Gonçalves, R., Pedrozo, A., Coutinho, E., Figueira, I., and Ventura, P. (2012). Efficacy of virtual reality exposure therapy in the treatment of PTSD: a systematic review. PLoS ONE 7:e48469. doi: 10.1371/journal.pone.0048469

Howard, M. (2017). A meta-analysis and systematic literature review of virtual reality rehabilitation programs. Comput. Hum. Behav. 70, 317-327. doi: 10.1016/j.chb.2017.01.013

In, T., Lee, K., and Song, C. (2016). Virtual reality reflection therapy improves balance and gait in patients with chronic stroke: randomized controlled trials. Med. Sci. Monit. 22, 4046-4053. doi: 10.12659/MSM.898157

Joong, H., Jang, S., Kim, C., Jung, J., and You, J. (2009). Use, of virtual reality to enhance balance and ambulation in chronic stroke: a doubleblind, randomized controlled study. Am. J. Phys. Med. Rehabil. 88, 693-701. doi: 10.1097/PHM.0b013e3181b33350

Kandalaft, M., Didehbani, N., Krawczyk, D., Allen, T., and Chapman, S. (2013). Virtual reality social cognition training for young adults with high-functioning autism. J. Autism Dev. Disord. 43, 34-44. doi: 10.1007/s10803-012-1544-6

Keshner, E., and Fung, J. (2017). The quest to apply VR technology to rehabilitation: tribulations and treasures. J. Vestib. Res. Equilib. Orient. 27, 1-5. doi: 10.3233/VES-170610

Kiefer, A. W., Rhea, C. K., and Warren, W. H. (2013). "Virtual reality based assessment and rehabilitation of functional mobility," in Human Walking in Virtual Environments: Perception, Technology, and Applications, eds F. Steinicke, Y. Visell, J. Campos, and A. Lécuyer (New York, NY: Springer), 333-350.

Killane, I., Fearon, C., Newman, L., McDonnell, C., Waechter, S., Sons, K., et al. (2015). Dual motor-cognitive virtual reality training impacts dual-task performance in freezing of gait. IEEE J. Biomed. Health Inform. 19, 1855-1861. doi: 10.1109/JBHI.2015.2479625 
Kim, J., Jang, S., Kim, C., , Jung, J., and You, J. (2009). Use of virtual reality to enhance balance and ambulation in chronic stroke: a doubleblind, randomized controlled study. Am. J. Phys. Med. Rehabil. 88, 693-701. doi: 10.1097/PHM.0b013e3181b33350

Lamontagne, A., Fung, J., McFadyen, B., and Faubert, J. (2007). Modulation of walking speed by changing optic flow in persons with stroke. J. Neuroeng. Rehabil. 4:22. doi: 10.1186/1743-0003-4-22

Latifi, R. (2008). Current Principles and Practices of Telemedicine and E-Health. Amsterdam: IOS Press.

Lee, I., Kim, Y., and Lee, D. (2015). Effect of a virtual reality exercise program accompanied by cognitive tasks on the balance and gait of stroke patients. J. Phys. Ther. Sci. 27, 2175-2177. doi: 10.1589/jpts.27.2175

Lee, K. (2015). Effects of a virtual reality-based exercise program on functional recovery in stroke patients: part 1. J. Phys. Ther. Sci. 27, 1637-1640. doi: 10.1589/jpts.27.1637

Li, A., Montaño, Z., Chen, V., and Gold, J. (2011). Virtual reality and pain management: current trends and future directions. Pain Manag. 1, 147-157. doi: $10.2217 /$ pmt.10.15

Liao, Y., Yang, Y., Cheng, S., Wu, Y., Fuh, J., and Wang, R. (2015). Virtual realitybased training to improve obstacle-crossing performance and dynamic balance in patients with Parkinson's disease. Neurorehabil. Neural Repair 29, 658-667. doi: $10.1177 / 1545968314562111$

Llorens, R., Noe, E., Colomer, C., and Alcaniz, M. (2015). Effectiveness, usability, and cost-benefit of a virtual reality-based telerehabilitation program for balance recovery after stroke: a randomized controlled trial. Arch. Phys. Med. Rehabil. 96, 418-425.e2. doi: 10.1016/j.apmr.2014.10.019

LoJacono, C., MacPherson, R., Kuznetsov, N., Raisbeck, L., Ross, S., and Rhea, C. (2017). Obstacle crossing in a virtual environment transfers to a real environment. J. Motor Learn. Dev. 6, 1-27. doi: 10.1123/jmld.2017-0019

Lyons, E., Lewis, Z., Mayrsohn, B., and Rowland, J. (2014). Behavior change techniques implemented in electronic lifestyle activity monitors: a systematic content analysis. J. Med. Internet Res. 16:e192. doi: 10.2196/jmir.3469

McEwen, D., Taillon-Hobson, A., Bilodeau, M., Sveistrup, H., and Finestone, H. (2014). Virtual reality exercise improves mobility after stroke: an inpatient randomized controlled trial. Stroke 45, 1853-1855. doi: 10.1161/STROKEAHA.114.005362

Michie, S., Abraham, C., Whittington, C., McAteer, J., and Gupta, S. (2009). Effective techniques in healthy eating and physical activity interventions: a meta-regression. Health Psychol. 28, 690-701. doi: 10.1037/a0016136

Michie, S., Johnston, M., Francis, J., Hardeman, W., and Eccles, M. (2008). From theory to intervention: mapping theoretically derived behavioural determinants to behaviour change techniques. Appl. Psychol. 57, 660-680. doi: 10.1111/j.1464-0597.2008.00341.x

Michie, S., Richardson, M., Johnston, M., Abraham, C., Francis, J., Hardeman, W., et al. (2013). The behavior change technique taxonomy (v1) of 93 hierarchically clustered techniques: building an international consensus for the reporting of behavior change interventions. Ann. Behav. Med. 46, 81-95. doi: 10.1007/s12160-013-9486-6

Middelweerd, A., Mollee, J., van der Wal, C., Brug, J., and Te Velde, S. (2014). Apps to promote physical activity among adults: a review and content analysis. Int. J. Behav. Nutr. Phys. Activity 11:97. doi: 10.1186/s12966-014-0097-9

Mirelman, A., Bonato, P., and Deutsch, J. (2009). Effects of training with a robotvirtual reality system compared with a robot alone on the gait of individuals after stroke. Stroke 40, 169-174. doi: 10.1161/STROKEAHA.108.516328

Mirelman, A., Maidan, I., Herman, T., Deutsch, J., Giladi, N., and Hausdorff, J. (2011). Virtual reality for gait training: can it induce motor learning to enhance complex walking and reduce fall risk in patients with Parkinson's disease. J. Gerontol. Ser. A Biol. Sci. Med. Sci. 66, 234-240. doi: 10.1093/gerona/glq201

Mirelman, A., Patritti, B., Bonato, P., and Deutsch, J. (2010). Effects of virtual reality training on gait biomechanics of individuals post-stroke. Gait Posture 31, 433-437. doi: 10.1016/j.gaitpost.2010.01.016

Moher, D., Shamseer, L., Clarke, M., Ghersi, D., Liberati, A., Petticrew, M., et al. (2015). Preferred reporting items for systematic review and meta-analysis protocols (PRISMA-P) 2015 statement. Syst. Rev. 4:1. doi: 10.1186/2046-4053-4-1

Molina, K., Ricci, N., de Moraes, S., and Perracini, M. (2014). Virtual reality using games for improving physical functioning in older adults: a systematic review. J. Neuroeng. Rehabil. 11:156. doi: 10.1186/1743-0003-11-156
Mulligan, H., Hale, L., Whitehead, L., and Baxter, G. (2012). Barriers to physical activity for people with long-term neurological conditions: a review study. Adapt. Phys. Activity Q. 29, 243-265. doi: 10.1123/apaq. 29.3.243

Opriş, D., Pintea, S., García-Palacios, A., Botella, C., Szamosközi, S., and David, D. (2012). Virtual reality exposure therapy in anxiety disorders: a quantitative meta-analysis. Depress. Anxiety 29, 85-93. doi: 10.1002/ da. 20910

Park, Y. H., Lee, C., and Lee, B. (2013). Clinical usefulness of the virtual realitybased postural control training on the gait ability in patients with stroke. $J$. Exerc. Rehabil. 9, 489-494. doi: 10.12965/jer.130066

Parsons, T., and Rizzo, A. (2008). Affective outcomes of virtual reality exposure therapy for anxiety and specific phobias: a meta-analysis. J. Behav. Ther. Exp. Psychiatry 39, 250-261. doi: 10.1016/j.jbtep.2007.07.007

Patel, K., Bailenson, J., Hack-Jung, S., Diankov, R., and Bajcsy, R. (2006). "The effects of fully immersive virtual reality on the learning of physical tasks," in Proceedings of the 9th Annual International Workshop on Presence (Cleveland, $\mathrm{OH}), 87-94$.

Pedreira da Fonseca, E., Ribeiro da Silva, N., and Pinto, E. (2017). Therapeutic effect of virtual reality on post-stroke patients: randomized clinical trial. J. Stroke Cerebrovasc. Dis. 26, 94-100. doi: 10.1016/j.jstrokecerebrovasdis.2016.08.035

Peruzzi, A., Zarbo, I., Cereatti, A., Croce, U., and Mirelman, A. (2017). An innovative training program based on virtual reality and treadmill: effects on gait of persons with multiple sclerosis. Disabil. Rehabil. 39, 1557-1563. doi: 10.1080/09638288.2016.1224935

Popescu, V., Burdea, G., Bouzit, M., and Hentz, V. (2000). A virtual-reality-based telerehabilitation system with force feedback. IEEE Trans. Inform. Technol. Biomed. 4, 45-51. doi: 10.1109/4233.826858

Porras, D., Siemonsma, P., Inzelberg, R., Zeilig, G., and Plotnik, M. (2018). Advantages of virtual reality in the rehabilitation of balance and gait: systematic review. Neurology 90, 1017-1025. doi: 10.1212/WNL.0000000000005603

Ravi, D., Kumar, N., and Singhi, P. (2017). Effectiveness of virtual reality rehabilitation for children and adolescents with cerebral palsy: an updated evidence-based systematic review. Physiotherapy 103, 245-258. doi: 10.1016/j.physio.2016.08.004

Rhea, C., and Kuznetsov, N. (2017). Using visual stimuli to enhance gait control. J. Vestib. Res. 27, 7-16. doi: 10.3233/VES-170602

Salem, Y., Gropack, S., Coffin, D., and Godwin, E. (2012). Effectiveness of a low-cost virtual reality system for children with developmental delay: a preliminary randomised single-blind controlled trial. Physiotherapy 98, 189-195. doi: 10.1016/j.physio.2012.06.003

Samdal, G., Eide, G., Barth, T., Williams, G., and Meland, E. (2017). Effective behaviour change techniques for physical activity and healthy eating in overweight and obese adults; systematic review and meta-regression analyses. Int. J. Behav. Nutr. Phys. Activity 14:42. doi: 10.1186/s12966-0170494-y

Schuler, T., Brutsch, K., Müller, R., van Hedel, H., and Meyer-Heim, A. (2011). Virtual realities as motivational tools for robotic assisted gait training in children: a surface electromyography study. NeuroRehabilitation 28, 401-411. doi: 10.3233/NRE-2011-0670

Singh, D., Nordin, N., Aziz, N., Lim, B., and Soh, L. (2013). Effects of substituting a portion of standard physiotherapy time with virtual reality games among community-dwelling stroke survivors. BMC Neurol. 13:199. doi: 10.1186/1471-2377-13-199

Song, G., and Park, E. (2015). Effect of virtual reality games on stroke patients balance, gait, depression, and interpersonal relationships. J. Phys. Ther. Sci. 27, 2057-2060. doi: 10.1589/jpts.27.2057

Steinicke, F., Visell, Y., Campos, J., and Lécuyer, A. (2013). Human Walking in Virtual Environments. New York, NY: Springer.

Teo, W., Muthalib, M., Yamin, S., Hendy, A., Bramstedt, K., Kotsopoulos, E., et al. (2016). Does a combination of virtual reality, neuromodulation and neuroimaging provide a comprehensive platform for neurorehabilitation? - a narrative review of the literature. Front. Hum. Neurosci. 10:284. doi: 10.3389/fnhum.2016.00284

van Gelder, L., Booth, A., van de Port, I., Buizer, A., Harlaar, J., and van der Krogt, M. (2017). Real-time feedback to improve gait in children with cerebral palsy. Gait Posture 52, 76-82. doi: 10.1016/j.gaitpost.2016.11.021 
Villiger, M., Bohli, D., Kiper, D., Pyk, P., Spillmann, J., Meilick, B., et al. (2013). Virtual reality-augmented neurorehabilitation improves motor function and reduces neuropathic pain in patients with incomplete spinal cord injury. Neurorehabil. Neural Repair 27, 675-683. doi: 10.1177/1545968313490999

Villiger, M., Liviero, J., Awai, L., Stoop, R., Pyk, P., Clijsen, R., et al. (2017). Home-based virtual reality-augmented training improves lower limb muscle strength, balance, and functional mobility following chronic incomplete spinal cord injury. Front. Neurol. 8:635. doi: 10.3389/fneur.2017.00635

Wall, T., Feinn, R., Chui, K., and Cheng, M. (2015). The effects of the nintendo wii fit on gait, balance, and quality of life in individuals with incomplete spinal cord injury. J. Spinal Cord Med. 38, 777-783. doi: 10.1179/2045772314Y.00000 00296

Wang, M., and Reid, D. (2011). Virtual reality in pediatric neurorehabilitation: attention deficit hyperactivity disorder, autism and cerebral palsy. Neuroepidemiology 36, 2-18. doi: 10.1159/000320847

Webb, T., Joseph, J., Yardley, L., and Michie, S. (2010). Using the internet to promote health behavior change: a systematic review and meta-analysis of the impact of theoretical basis, use of behavior change techniques, and mode of delivery on efficacy. J. Med. Internet Res. 12:e4. doi: 10.2196/ jmir.1376

Yang, C., Maher, J., and Conroy, D. (2015). Implementation of behavior change techniques in mobile applications for physical activity. Am. J. Prev. Med. 48, 452-455. doi: 10.1016/j.amepre.2014.10.010
Yang, Y., Tsai, M., Chuang, T., Sung, W., and Wang, R. (2008). Virtual reality-based training improves community ambulation in individuals with stroke: a randomized controlled trial. Gait Posture 28, 201-206. doi: 10.1016/j.gaitpost.2007.11.007

Yom, C., Cho, H., and Lee, B. (2015). Effects of virtual reality-based ankle exercise on the dynamic balance, muscle tone, and gait of stroke patients. J. Phys. Ther. Sci. 27, 845-849. doi: 10.1589/jpts.27.845

Zimmerli, L., Jacky, M., Lunenburger, L., Riener, R., and Bolliger, M. (2013). Increasing patient engagement during virtual reality-based motor rehabilitation. Arch. Phys. Med. Rehabil. 94, 1737-1746. doi: 10.1016/j.apmr.2013.01.029

Conflict of Interest Statement: The authors declare that the research was conducted in the absence of any commercial or financial relationships that could be construed as a potential conflict of interest.

Copyright (C) 2019 Felsberg, Maher and Rhea. This is an open-access article distributed under the terms of the Creative Commons Attribution License (CC BY).

The use, distribution or reproduction in other forums is permitted, provided the original author(s) and the copyright owner(s) are credited and that the original publication in this journal is cited, in accordance with accepted academic practice. No use, distribution or reproduction is permitted which does not comply with these terms. 